\title{
ON THE INVERTIBILITY OF GENERAL WIENER-HOPF OPERATORS
}

\author{
JOHN REEDER
}

\begin{abstract}
Let $\mathfrak{G}$ be a separable Hilbert space, $\mathfrak{B}$ the set of bounded linear operators on $\mathfrak{E}$, and $P$ an orthogonal projection on $\mathfrak{W}$. Denote the range of $P$ by $R(P)$. Let $A$ belong to $\mathfrak{B}$. The general Wiener-Hopf operator associated with $A$ and $P$ is defined by $T_{P}(A)=P A \mid R(P)$, the vertical bar denoting restriction. Let $Q=I-P$. The purpose of this paper is to disprove the general conjecture that if $A$ is an invertible element of $\mathfrak{B}$, then the invertibility of $T_{P}(A)$ implies the invertibility of $T_{Q}(A)$. We also disprove the conjecture in an interesting special case.
\end{abstract}

1. Introduction. Let $\mathfrak{S}$ be a separable Hilbert space, and $\mathfrak{B}$ the set of bounded linear operators on $\mathfrak{S}$. Let $P$ be an orthogonal projection on $\mathfrak{S}$. Denote its range by $R(P)$. Let $A$ belong to $\mathfrak{B}$. The general Wiener-Hopf operator associated with $A$ and $P$ is defined by

$$
T_{P}(A)=P A \mid R(P),
$$

the vertical bar denoting restriction. Let $Q=I-P$. The purpose of this paper is to disprove the general conjecture that if $A$ is an invertible element of $\mathfrak{B}$, then the invertibility of $T_{P}(A)$ implies the invertibility of $T_{Q}(A)$. We also disprove the conjecture in an interesting special case.

In $\$ 2$ we mention some special cases in which the conjecture has been proven true. We then exhibit a simple counterexample to prove that the conjecture is, in general, false.

In order to discuss $\$ 3$ we introduce some additional terminology. Let $L^{2}(T ; \mathfrak{S})$ denote the space of equivalence classes of $\mathfrak{S}$-valued, weakly measurable functions on the circle group $T$ which are square summable; $H^{2}(T ; \mathfrak{S})$ the subspace of $L^{2}(T ; \mathfrak{W})$ which consists of those elements whose Fourier coefficients vanish on the negative integers; and $K^{2}(T ; \mathfrak{S})$ the orthogonal complement of $H^{2}(T ; \mathfrak{S})$ in $L^{2}(T ; \mathfrak{S}) . P$ and $Q$ are projections from $L^{2}(T ; \mathfrak{S})$ onto $H^{2}(T ; \mathfrak{W})$ and $K^{2}(T ; \mathfrak{S})$ respectively. All operators are assumed to be bounded and linear. Let $A(\theta)$ be any weakly measurable, essentially bounded, $\mathfrak{B}$ valued function on the circle group. We define an operator $A$ on $L^{2}(T ; \mathfrak{S})$ by

Received by the editors January 16, 1970 and, in revised form, April 23, 1970.

AMS 1969 subject classifications. Primary 4615, 4690; Secondary 4725.

Key words and phrases. General Wiener-Hopf operators. 


$$
(A f)(\theta)=A(\theta) f(\theta), \quad f \in L^{2}(T ; \mathfrak{W}), \quad \theta \in T,
$$

and two Wiener-Hopf operators by

$$
T_{P}(A)=P A\left|H^{2}(T ; \mathfrak{W}), \quad T_{Q}(A)=Q A\right| K^{2}(T ; \mathfrak{S}) .
$$

As usual, $\mathbb{E}$ denotes the complex numbers. Finally, suppose that the operator $A$ is invertible.

Devinatz and Shinbrot [1] have shown that if $H$ is one-dimensional, then $T_{P}(A)$ being invertible implies $T_{Q}(A)$ is invertible. They remark: "Whether or not this result persists when the dimension of $\mathfrak{E}$ is greater than 1 is not known." (The remark follows proof of Corollary 8, $\$ 6$ in [1].) We shall prove that the result does not by exhibiting a counterexample when the dimension of $\mathfrak{S}$ is two (i.e. $\mathfrak{S}=\mathfrak{C} \times \mathfrak{C}$ ). The counterexample is of the form $U^{*} B U$ where $U$ is a certain unitary operator on $L^{2}\left(T ; \mathfrak{E} \times(\mathfrak{E})\right.$ mapping $H^{2}\left(T ; \mathfrak{E} \times(\mathfrak{E})\right.$ onto $L^{2}(T ; \mathfrak{E}) \times\{0\}$ and $K^{2}(T ; \mathfrak{E} \times \mathbb{E})$ onto $\{0\} \times L^{2}(T ; \mathfrak{E})$, and $B$ is an invertible operator on $L^{2}(T ; \mathbb{E} \times \mathbb{E})$ patterned after the counterexample of $\S 2$. This counterexample may be generalized to disprove the result when the dimension of $\mathfrak{S}$ is any integer greater than one.

2. The first counterexample. Devinatz and Shinbrot have proven that for a unitary operator $U$, the invertibility of $T_{P}(U)$ is equivalent to that of $T_{Q}(U)$ for any orthogonal projection $P$ on $\mathfrak{S}$ where $Q=I-P$ (Corollary $1, \S 2$ in [1]). They have also shown that if $A$ is any operator with a strongly positive real part $(\operatorname{Re}(A x, x) \geqq \delta(x, x), x \in \mathfrak{S}, \delta$ positive), then $T_{P}(A)$ is invertible for every orthogonal projection $P$. In particular, it is invertible for $Q=I-P$ (Lemma 2, \$2 in [1]). Pellegrini proved that for an operator $A, T_{P}(A)$ is invertible for every orthogonal projection if and only if there exists a $\theta$ between 0 and $2 \pi$ such that $e^{i \theta} A$ has strongly positive real part (Theorem 1.2.10 in [2]). The following simple counterexample illustrates that the invertibility of $T_{P}(A)$ does not guarantee that of $T_{Q}(A)$, even if $A$ is invertible.

Counterexample 1. Take $\mathfrak{S}=\mathfrak{E} \times \mathfrak{E}, P$ the projection from $\mathfrak{E}$ onto $\mathfrak{S} \times\{0\}$, and $Q=I-P$. Let $A$ be the matrix $\left(\begin{array}{l}1 \\ 1\end{array} 0\right)$. The determinant of $A$ is -1 so that $A$ is invertible. It is easily seen that $T_{P}(A)=I \mid \mathbb{E} \times\{0\}$ and $T_{Q}(A)=0 \mid\{0\} \times \mathbb{E}$.

3. The second counterexample. In this section $P$ is the projection from $L^{2}(T ; \mathfrak{E} \times \mathfrak{E})$ onto $H^{2}(T ; \mathfrak{E} \times \mathfrak{E})$ and $Q=I-P ; P^{\prime}$ is the projection from $L^{2}(T ; \mathfrak{E} \times \mathbb{E})$ onto $L^{2}(T, \mathfrak{E}) \times\{0\}$ and $Q^{\prime}=I-P^{\prime}$. We shall construct an invertible operator $A$ on $L^{2}(T ; \mathfrak{E} \times \mathbb{E})$ such that $T_{P}(A)$ is invertible but $T_{Q}(A)$ is not. 
Let $[$,$] denote the inner product in both \mathfrak{E} \times \mathfrak{F}$ and $\mathfrak{E} ;\langle$,$\rangle the$

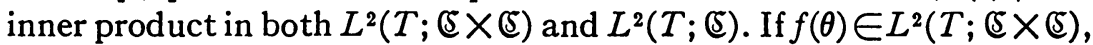
then $f$ is easily seen to be of the form $\left(f_{1}(\theta), f_{2}(\theta)\right)$ where $f_{1}$, $f_{2} \in L^{2}\left(T ;(\mathbb{C})\right.$. Let $g(\theta)=\left(g_{1}(\theta), g_{2}(\theta)\right)$ be another element of $L^{2}(T ;(\mathbb{E} \times \mathbb{(})$. The topology on $L^{2}(T ; \mathfrak{E} \times \mathbb{E})$ is determined by the inner product:

$$
\langle f, g\rangle=\int_{\theta \in T}[f(\theta), g(\theta)] d \theta=\int_{\theta \in T}\left[\left(f_{1}(\theta), f_{2}(\theta)\right),\left(g_{1}(\theta), g_{2}(\theta)\right)\right] d \theta .
$$

We define inner product on $\mathfrak{E} \times(\mathfrak{S}$ to be the sum of the respective inner products. Thus, (3.1) becomes

$$
\begin{aligned}
\langle f, g\rangle & =\int_{\theta \in T}\left[f_{1}(\theta), g_{1}(\theta)\right] d \theta+\int_{\theta \in T}\left[f_{2}(\theta), g_{2}(\theta)\right] d \theta \\
& =\left\langle f_{1}, g_{1}\right\rangle+\left\langle f_{2}, g_{2}\right\rangle .
\end{aligned}
$$

This indicates that we may identify $L^{2}(T ; \mathfrak{E} \times \mathbb{E})$ with $L^{2}(T ; \mathfrak{E})$ $\times L^{2}(T ; \mathfrak{E})$ where the inner product in the latter space is given by the right-hand side of (3.2). Under this identification, $H^{2}(T ; \mathfrak{E} \times \mathbb{E})$ and $K^{2}\left(T ; \mathfrak{E} \times(\mathfrak{S})\right.$ become $H^{2}\left(T\right.$; $\mathfrak{( S )} \times H^{2}\left(T ;(\mathfrak{S})\right.$ and $K^{2}\left(T ;(\mathfrak{S}) \times K^{2}(T ; \mathfrak{E})\right.$ respectively. We make this identification freely throughout the remainder of the paper.

The Plancherel theorem says that the Fourier transform, $F$, is an isometry from $L^{2}\left(T ;(\mathfrak{S})\right.$ onto $l^{2}$, the square-summable, (5-valued sequences on the integers. The adjoint of $F, F^{*}$, is easily seen to equal $F^{-1}$. We make use of these facts in the following lemma.

Lemma 1. There exists a unitary operator $U$ on $L^{2}(T$; $\mathfrak{E} \times \mathfrak{(})$ such that

(i) $U=U^{*}=U^{-1}$,

(ii) $U\left(H^{2}\left(T ;(\mathbb{E} \times(\mathfrak{S}))=L^{2}(T\right.\right.$; $\mathfrak{(}) \times\{0\}$,

(iii) $U\left(K^{2}(T ; \mathfrak{E} \times \mathbb{E})\right)=\{0\} \times L^{2}(T ; \mathfrak{E})$.

Proof. For $\left(f_{n}\right),\left(g_{n}\right) \in l^{2}$, define a map $G: l^{2} \times l^{2} \rightarrow l^{2} \times l^{2}$ by

$$
G\left(\left(f_{n}\right),\left(g_{n}\right)\right)=\left(\left(h_{n}\right),\left(k_{n}\right)\right)
$$

where

$$
\begin{aligned}
h_{n} & =f_{n}, & & n \geqq 0, \\
& =g_{-n-1}, & & n<0, \\
k_{n} & =f_{-n-1}, & & n \geqq 0, \\
& =g_{n}, & & n<0 .
\end{aligned}
$$




$$
\begin{aligned}
\left\|G\left(\left(f_{n}\right),\left(g_{n}\right)\right)\right\|^{2} & =\sum f_{n} \bar{f}_{n}+\sum g_{-n-1} \bar{g}_{-n-1}+\sum f_{-n-1} \bar{f}_{-n-1}+\sum g_{n} \bar{g}_{n} \\
& =\left\|\left(f_{n}\right)\right\|^{2}+\left\|\left(g_{n}\right)\right\|^{2} .
\end{aligned}
$$

The inner product in $l^{2} \times l^{2}$ is taken to be the sum of the respective inner products in $l^{2}$, so that $\left\|\left(\left(f_{n}\right),\left(g_{n}\right)\right)\right\|^{2}=\left\|\left(f_{n}\right)\right\|^{2}+\left\|\left(g_{n}\right)\right\|^{2}$. Observe that $G$ is linear. Hence $G$ is an isometry. Note that $G^{2}=I$, so that $G=G^{-1}$. Let $h^{2} \times h^{2}\left(k^{2} \times k^{2}\right)$ denote the subspace of $l^{2} \times l^{2}$ of series whose terms vanish for negative (positive) indices. Observe that $G$ maps $h^{2} \times h^{2}$ onto $l^{2} \times\{0\}$ and $k^{2} \times k^{2}$ onto $\{0\} \times l^{2}$.

Now we define a map $H: L^{2}\left(T ;(\mathfrak{S}) \times L^{2}\left(T ;(\mathfrak{S}) \rightarrow l^{2} \times l^{2}\right.\right.$ via the Fourier transform, $F$, as follows

$$
\begin{gathered}
H(f, g)=(F(f), F(g)), \quad(f, g) \in L^{2}\left(T ;(\mathfrak{E}) \times L^{2}(T ;(\mathfrak{E}),\right. \\
\|H(f, g)\|^{2}=\|F(f)\|^{2}+\|F(g)\|^{2}=\|f\|^{2}+\|g\|^{2} .
\end{gathered}
$$

Note that $H$ is linear because $F$ is linear. Hence $H$ is an isometry. It takes $H^{2}\left(T\right.$; () $\times H^{2}(T$; () $)$ onto $h^{2} \times h^{2}, K^{2}\left(T\right.$; (E) $\times K^{2}(T$; (E) onto $k^{2} \times k^{2}, L^{2}(T ; \mathfrak{E}) \times\{0\}$ onto $l^{2} \times\{0\}$, and $\{0\} \times L^{2}(T ;(\mathfrak{E})$ onto $\{0\}$ $\times l^{2}$. It is easily seen that $H^{*}=H^{-1}$.

Finally, we set $U=H^{-1} G H$. If we make the identification of $L^{2}\left(T\right.$; $\sqrt{ } \times(\mathfrak{S})$ with $L^{2}(T$; $\sqrt{ }) \times L^{2}(T$; $\sqrt{ })$ mentioned earlier, then $U$ may be considered as an operator taking $L^{2}(T$; $\mathbb{E} \times \mathbb{E})$ onto itself. Now (ii) and (iii) follow immediately from the above mentioned properties of $G$ and $H$. We prove (i):

$$
U^{*}=H^{*} G^{*}\left(H^{-1}\right)^{*}=H^{-1} G H=U, \quad U^{-1}=H^{-1} G^{-1} H=H^{-1} G H=U .
$$

The following operator is central to the forthcoming counterexample. Its construction is patterned after that of Counterexample 1. Let

$$
\begin{gathered}
B(\theta)=\left(\begin{array}{ll}
b_{11}(\theta) & b_{12}(\theta) \\
b_{21}(\theta) & b_{22}(\theta)
\end{array}\right) \\
\text { where }\left\{\begin{array}{l}
b_{11}(\theta)=b_{12}(\theta)=b_{21}(\theta)=1, \\
b_{22}(\theta)=0,
\end{array} \quad \theta \in T .\right.
\end{gathered}
$$

Define

$$
(B f)(\theta)=B(\theta) f(\theta), \quad f \in L^{2}(T ; \mathfrak{E} \times(\mathfrak{E}), \quad \theta \in T .
$$

The properties of $B$ which will be of interest to us are contained in the following lemma.

LEMMA 2. $B$ is an invertible operator on $L^{2}(T ; \mathfrak{E} \times(\mathfrak{E})$ such that

(i) $T_{P^{\prime}}(B)=I \mid L^{2}(T ;$ ( $) \times\{0\}$,

(ii) $T_{Q^{\prime}}(B)=0 \mid\{0\} \times L^{2}(T ;$ ( $)$. 
Proof. For any $f \in L^{2}(T ; \mathfrak{E} \times \mathbb{E})$ we have shown that $f=\left(f_{1}, f_{2}\right)$ where $f_{1}, f_{2} \in L^{2}\left(T\right.$; $(\mathcal{E})$. By (3.3), $B\left(f_{1}, f_{2}\right)=\left(f_{1}+f_{2}, f_{1}\right)$. Hence (i) and (ii) follow immediately. It is easily seen that $B^{-1}\left(f_{1}, f_{2}\right)=\left(f_{2}, f_{1}-f_{2}\right)$. The fact that $B$ and $B^{-1}$ are bounded and linear is also easily shown.

The following simple lemma is proven in a general context.

Lemma 3. Let $U$ be a unitary operator on a Hilbert space $\mathfrak{g}$. Suppose $P$ and $P^{\prime}$ are two orthogonal projections on $\mathfrak{S}$ such that $U(R(P)$ ) $=R\left(P^{\prime}\right)$ and $U(R(Q))=R\left(Q^{\prime}\right)\left(Q=I-P, Q^{\prime}=I-P^{\prime}\right)$. Let $B$ belong to 28. Then

(i) $T_{P}\left(U^{*} B U\right)=U^{*} T_{P^{\prime}}(B) U \mid R(P)$,

(ii) $T_{Q}\left(U^{*} B U\right)=U^{*} T_{Q^{\prime}}(B) U \mid R(Q)$.

Proof. First we note that $\left(U^{*} P^{\prime} U\right)^{2}=U^{*} P^{\prime} U,\left(U^{*} P^{\prime} U\right)^{*}=U^{*} P^{\prime} U$, and $R\left(U^{*} P^{\prime} U\right)=R(P)$. Hence $P=U^{*} P^{\prime} U$. The rest is easy:

$$
\begin{aligned}
U^{*} T_{P^{\prime}}(B) U \mid R(P) & =U^{*}\left(P^{\prime} U U^{*} B \mid R\left(P^{\prime}\right)\right) U \mid R(P) \\
& =P\left(U^{*} B U\right) \mid R(P)=T_{P}\left(U^{*} B U\right) .
\end{aligned}
$$

The proof for $T_{Q}\left(U^{*} B U\right)$ is identical.

We are now prepared to exhibit the following counterexample.

CoUNTEREXAMPLE 2. There exists an invertible operator $A$ on $L^{2}(T ; \mathfrak{E} \times \mathbb{E})$ such that $T_{P}(A)=I \mid H^{2}(T ; \quad \mathfrak{E} \times \mathfrak{E})$ and $T_{\mathbb{Q}}(A)=$ $0 \mid K^{2}(T ; \mathfrak{E} \times \mathfrak{E})$.

Proof. Let $U$ be the operator of Lemma 1; $B$, that of Lemma 2; and set $A=U^{*} B U$. By Lemmas 1 and 2, $A$ is an invertible operator on $L^{2}(T ; \mathfrak{(} \times(\mathfrak{S})$. Moreover, Lemma 3 says that

$$
U^{*} T_{P^{\prime}}(B) U \mid H^{2}(T ; \sqrt{ } \times \sqrt{ })=T_{P}\left(U^{*} B U\right) .
$$

But $T_{P^{\prime}}(B)=I \mid L^{2}(T ;$ ( $) \times\{0\}$ by (i) of Lemma 2. Thus, using (i) and (ii) of Lemma 1, we get that

$$
T_{P}(A)=T_{P}\left(U^{*} B U\right)=I \mid H^{2}(T ; \mathfrak{c} \times(\mathfrak{c}) .
$$

Similarly, one sees that

$$
T_{\mathbb{Q}}(A)=T_{Q}\left(U^{*} B U\right)=U^{*} T_{Q^{\prime}}(B) U\left|K^{2}(T ; \mathfrak{E} \times \mathbb{E})=0\right| K^{2}(T ; \mathfrak{E} \times \mathbb{E}) .
$$

\section{REFERENCES}

1. A. Devinatz and M. Shinbrot, General Wiener-Hopf operators, Trans. Amer. Math. Soc. 145 (1969), 467-494.

2. V. J. Pellegrini, Wiener-Hopf operators, Ph.D. Thesis, Northwestern University, Evanston, Ill.

NORTHWESTERN UNIVERSITy, Evanston, IllinoIs 60201 